\title{
Thyroid Gland Metastasis from Cancer of the Uterine Cervix: An Extremely Rare Case Report
}

\author{
Suleyman Utku Celik ${ }^{1, *}$, Dilara Besli', Serpil Dizbay Sak', Volkan Genc ${ }^{1}$ \\ ${ }^{1}$ Department of General Surgery, Ankara University School of Medicine, Ankara, Turkey \\ ${ }^{2}$ Department of Pathology, Ankara University School of Medicine, Ankara, Turkey \\ * Corresponding author: Ankara University School of Medicine, Ibn-i Sina Hospital, 06100, Sihhiye, Ankara, Turkey; e- \\ mail: s.utkucelik@hotmail.com
}

\begin{abstract}
Summary: The thyroid gland is a relatively uncommon site for a metastatic disease, although it is richly supplied with blood. The metastases may originate from various primary sites, mainly kidney, lung, head and neck, and breast. Thyroid metastasis from cervical carcinomas is extremely rare; and only a few cases have been previously reported in the literature. In patient with thyroid nodules and an oncological history, the possibility of thyroid metastasis should be seriously considered. Despite the rarity of the metastasis of cervical carcinoma to the thyroid, it is difficult to say appropriate treatment approach for these lesions. When managing such patients, decision-making should balance the possibility of gaining longterm survival against estimation of the aggressiveness of the disease and its possible complications. Here, a case of thyroid metastasis from a squamous cell carcinoma of the uterine cervix presenting with cervical mass and difficulty in swallowing and its treatment is reported.
\end{abstract}

Keywords: Metastasis; Thyroid gland; Cancer of the uterine cervix

\section{Introduction}

Worldwide, uterine cervix cancer is the most common gynecologic malignancy among women with an estimated 527,600 new cases in 2012 and tends to have a favorable prognosis $(1,2)$. It spreads predominantly by direct local extension and lymphatic metastasis. The most common metastatic sites are the vagina, parametrium, and pelvic lymph nodes (3). Metastasis of cervical carcinoma to the thyroid is extremely rare and only a few cases have been previously reported (3-5).

Although the thyroid gland has a rich blood supply, clinically detectable tumor metastases to the thyroid are uncommon, accounting for approximately $1.4 \%$ to $3 \%$ of all thyroid malignancies $(4,6)$. Moreover, numerous large autopsy series have reported an incidence ranging from $1.9 \%$ to $24 \%$ in patients who died due to malignancies at other primary sites (7). Herein, we report a case of thyroid metastasis from a squamous cell carcinoma of the cervix presenting with painless cervical mass and difficulty in swallowing and its treatment in a 56-year-old woman.

\section{Case report}

A 56-year-old woman with a six-month history of uterine cervical squamous cell carcinoma treated with total abdomi- nal hysterectomy and bilateral salpingo-oophorectomy plus combining adjuvant radiotherapy and chemotherapy was admitted to our clinic with bilateral palpable masses on the thyroid. The complaints of the patient were swelling on the neck and severe difficulty in swallowing. On physical examination, a diffusely enlarged thyroid and a $3-\mathrm{cm}$ moderately hard nodule were detected in the right lobe without palpable cervical lymphadenopathy. The other systemic evaluation was unremarkable except for mild hypertension. The patient had no other significant past medical history and no family history.

Preoperative thyroid function tests showed the following results: free triiodothyronine $\left(\mathrm{fT}_{3}\right): 5.6 \mathrm{pmol} / \mathrm{L}(3.8-6)$, free thyroxine $\left(\mathrm{fT}_{4}\right): 13.9 \mathrm{pmol} / \mathrm{L}(7-16)$, and thyroid-stimulating hormone (TSH): $0.02 \mu \mathrm{IU} / \mathrm{mL}(0.34-5.6)$. Thyroglobulin: $128 \mathrm{ng} / \mathrm{mL}(1.4-78)$, anti-Tg: $1.6 \mathrm{IU} / \mathrm{mL}(0-4)$, and anti-TPO: $0.25 \mathrm{IU} / \mathrm{mL}(0-9)$. Ultrasonographic thyroid examination revealed bilateral and multiple nodules with micro- and macrocalcifications that the largest was heterogeneous, hypoechoic, and approximately with a diameter of $34 \mathrm{~mm}$ in right lobe. An ${ }^{18} \mathrm{~F}$-fluorodeoxyglucose (FDG)-positron emission tomography (PET/CT) scan was performed to further evaluate any possible sites of metastatic disease. The whole body ${ }^{18} \mathrm{~F}$ - FDG PET/CT scan demonstrated significant uptake in the right upper lobe of the lung, lumbar spine, paraaortic and paratracheal lymph nodes. Multiple bilater- 


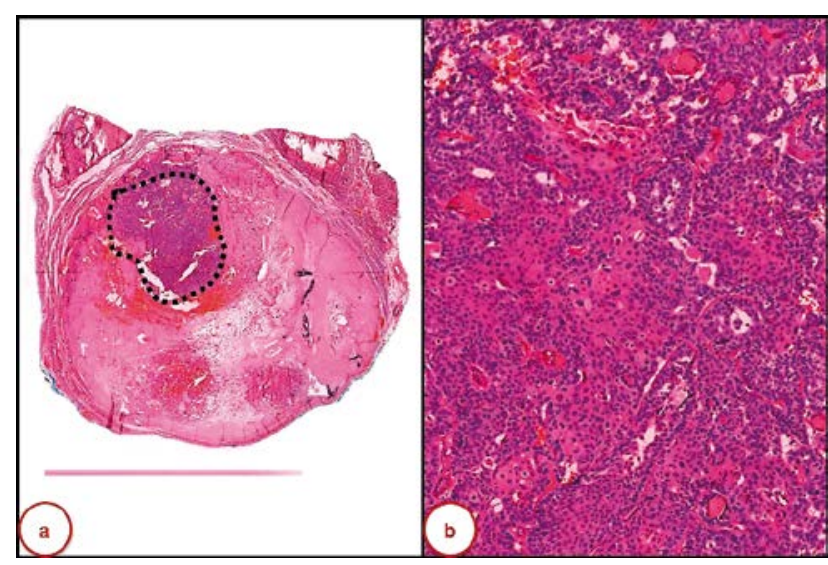

Fig. 1: (a) A well demarcated area (dotted) of metastatic tumor was observed within a benign nodule $(0.6 \times, \mathrm{HE})$; (b) Tumor islands were composed of atypical squamous cells $(15.2 \times, \mathrm{HE})$.

al thyroid nodules were also seen with no hypermetabolic activity and the biggest one, which was in the right lower pole, was calcified and about 3 centimeters in diameter as well.

In view of the rapid growth and progressing symptoms of dysphagia and difficulty in swallowing, palliative surgery was planned and the patient subsequently underwent total thyroidectomy and central lymph node dissection. Macroscopically, multiple nodules were observed in both lobes and isthmus, the largest located in the right lobe measuring $32 \mathrm{~mm}$ in diameter and showing hemorrhagic areas. On histopathological examination, multiple benign nodules were observed. On the right lobe, two areas measuring $3 \mathrm{~mm}$ and $10 \mathrm{~mm}$ were noted, within two different thyroid nodules (Figure 1). These areas were observed as sharply demarcated foci, within benign thyroid nodules, showing islands of atypical mitotically active squamous cells. Immunohistochemical staining revealed positive status for cytokeratin 5/6, cytokeratin 19, CEA, HBME-1, galectin-3, p63, and estrogen receptor whereas negative for thyroglobulin, thyroid transcription factor-1 (TTF-1), progesterone receptor, calcitonin, synaptophysin, CD56, and chromogranin A in these foci (Figure 2). p53 and Ki-67 were expressed in 5\% and $30 \%$ of tumor cell nuclei, respectively. In situ hybridization for human papilloma virus was also negative. These foci were considered as metastasis from cervical squamous cell carcinoma. The patient was discharged in good general condition on postoperative day 2 . The clinical status of the patient rapidly deteriorated and she died five months after the diagnosis of thyroid metastasis.

\section{Discussion}

Clinically evident metastases to the thyroid gland are generally considered rare, commonly associated with concurrent metastatic lesions to other organs and usually indicate a poor prognosis. Despite the high prevalence in

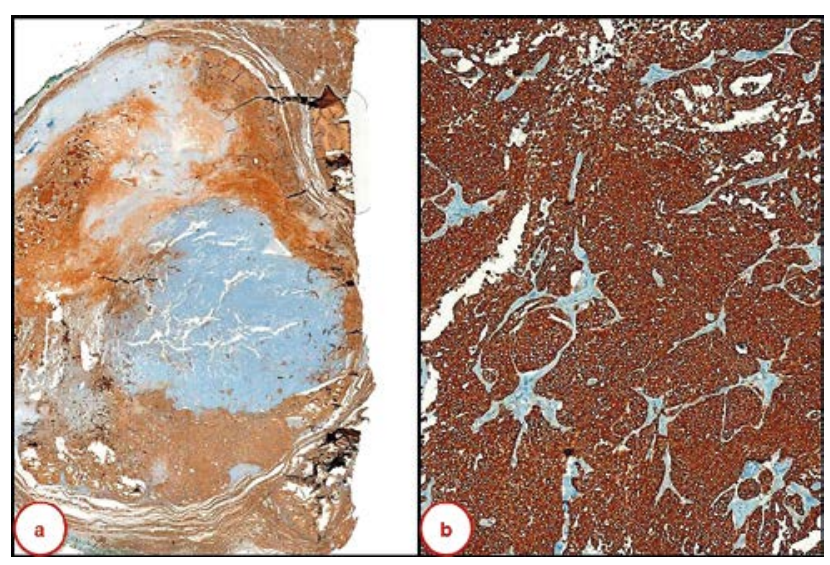

Fig. 2: (a) Tumor cells were negative by thyroglobulin immunostaining $(0.8 \times$, thyroglobulin); (b) Tumor cells were strongly CK $5 / 6$ positive $(8.6 \times, \mathrm{CK} 5 / 6)$.

autopsy series, metastases represent approximately $1.4 \%$ to $3 \%$ of all malignant tumors of the thyroid $(6,7)$; possibly due to a high concentration of oxygen and iodine in the thyroid tissue which may impair the ability of metastatic cells to settle and develop (8). The high intrathyroidal vascular flow also probably plays a role in impeding the adhesion and implantation of metastatic tumor emboli (9).

Recently, Hegerova et al. identified 97 patients with a metastatic solid neoplasm of the thyroid gland. In this analysis, $22 \%$ of cases were metastases from renal cancer, $22 \%$ from lung, 12\% from head and neck, $11 \%$ were from breast cancer, and 9\% from esophagus (6). Less commonly, metastases originated from skin, neuroendocrine, and ovary/ uterus. Thyroid metastasis from uterine cervical squamous cell carcinomas is extremely rare; and to the best of our knowledge, only a few cases have been previously reported in English-language literature (3-5). The median and mean age at discovery of clinically significant metastases to the thyroid are 63 and 59 years, with a female predominance $(6,10)$. The mean latency between diagnosing primary malignancy and its metastases to the thyroid gland is variable 53 to 70 months $(6,10)$. The mean interval between diagnosing primary tumor and discovering its metastases to thyroid is longest in patients with sarcomas (75 months) and shortest in lung cancer (4.5 months) (10). In the present case, the latency was about six months.

The majority of patients with a thyroid metastasis present with an asymptomatic thyroid nodule and it is difficult to determine whether the tumor is primary or secondary. Neither ultrasonography nor scintigraphy provides specific data as cold nodules on radioiodine uptake studies or as a heterogeneous and hypoechoic mass on ultrasonographic examination (9). Thyroid function tests usually show no abnormalities and most patients are euthyroid (87.6\%). Occasionally, some patients may develop a transient changes in thyroid hormones, so called non-thyroidal illness or euthyroid sick syndrome (10). The laboratory parameters of 
this condition characterized mainly by low serum $\mathrm{fT}_{3}$, with normal or low $\mathrm{fT}_{4}$ and normal or suppressed levels of TSH. With the advanced diagnostic imaging technology such as ${ }^{18} \mathrm{~F}$ - FDG PET/CT, an increasing number of incidental cases of metastatic disease to the thyroid gland are likely to be detected (8). Fine-needle aspiration cytology is able to make the differentiation between benign and malignant thyroid diseases with a high negative predictive value, high specificity (100\%), and sensitivity (94\%) and help avoid unnecessary surgery $(6,7,9)$. Immunohistochemically, negative staining with anti-thyroglobulin and anti-calcitonin antibodies would favor a metastatic tumor. Moreover, in patients with an oncological history of presenting with thyroid nodules, the possibility of thyroid metastasis should always be considered (8). Patients with previously known multinodular goiter and follicular adenoma have also been associated with an increased incidence of metastases to thyroid (8). In the present case, metastatic tumor deposits occupied only a small proportion of the benign thyroid nodules.

There is no clear consensus about the surgical procedure that should be performed in patients with thyroid metastases (9). To date, however, no conclusive survival advantage following surgery has been proven for metastases to the thyroid gland which represent advanced systemic disease $(11,12)$. In most cases, it is suggested that metastases to the thyroid are associated with a poor prognosis. Although controversial, radical treatment for isolated metastases can be curative and an aggressive surgical approach has been recommended by many authors $(6,7,9)$. For other patients with metastatic neoplasm of the thyroid gland which manifestation of a widely metastatic disease, the aim of the surgery must be palliative such as the prevention of asphyxia and other local complications (9). Adjuvant chemotherapy and/or radiotherapy may be considered as palliative treatment for local control of the disease. In our reported case, because of the tumors rapid growing with possible airway compression palliative surgery was planned and the patient subsequently underwent total thyroidectomy.

In conclusion, although metastatic diseases to the thyroid gland are uncommon, it is important for the pathologists, endocrine surgeons, and oncologists to be able to recognize and make the differentiation between the other more common thyroid malignancies. Due to the rarity of these lesions it is difficult to determine the survival advantage of surgical intervention for patients with metastasis to the thyroid. Because of metastatic thyroid tumor from uterine cervix carcinoma is extremely rare, the decision-making should balance the possibility of gaining long-term survival against the risk of complications, worsening the outcome and decrease in quality of life. It should be noted that most of the thyroid metastases present in the context of widespread metastatic disease and the metastatic diseases to the thyroid tend to behave more aggressively.

\section{References}

1. Torre LA, Bray F, Siegel RL, Ferlay J, Lortet-Tieulent J, Jemal A. Global cancer statistics, 2012. CA Cancer J Clin 2015; 65: 87-108.

2. Stany MP, Stone PJ, Felix JC, et al. Lymph node micrometastases in early-stage cervical cancer are not predictive of survival. Int J Gynecol Pathol 2015; 34: 379-84.

3. Karapanagiotou E, Saif MW, Rondoyianni D, et al. Metastatic cervical carcinoma to the thyroid gland: a case report and review of the literature. Yale J Biol Med 2006; 79: $165-8$

4. Vamsy M, Dattatreya PS, Sarma LY, Dayal M, Janardhan N, Rao VV. Metastatic squamous cell carcinoma thyroid from functionally cured cancer cervix. Indian J Nucl Med 2013; 28: 112-4.

5. Martino E, Bevilacqua G, Nardi M, Macchia E, Pinchera A. Metastatic cervical carcinoma presenting as primary thyroid cancer. Case report. Tumori 1977; 63: $25-30$.

6. Hegerova L, Griebeler ML, Reynolds JP, Henry MR, Gharib H. Metastasis to the thyroid gland: report of a large series from the Mayo Clinic. Am J Clin Oncol 2015; 38: $338-42$.

7. Wood K, Vini L, Harmer C. Metastases to the thyroid gland: the Royal Marsden experience. Eur J Surg Oncol 2004; 30: 583-8.

8. Cherk MH, Moore M, Serpell J, Swain S, Topliss DJ. Metastatic colorectal cancer to a primary thyroid cancer. World J Surg Oncol 2008; 6: 122.

9. Montero PH, Ibrahimpasic T, Nixon IJ, Shaha AR. Thyroid metastasectomy. J Surg Oncol 2014; 109: 36-41.

10. Chung AY, Tran TB, Brumund KT, Weisman RA, Bouvet M. Metastases to the thyroid: a review of the literature from the last decade. Thyroid 2012; 22: 258-68.

11. Park MH, Cho JS, Lee JS, Kim HK, Yoon JH. Thyroid gland metastasis arising from primary liver cholangiocarcinoma: The first case report involving surgical operation. Int J Surg Case Rep 2012; 3: 78-81.

12. Nixon IJ, Whitcher M, Glick J, et al. Surgical management of metastases to the thyroid gland. Ann Surg Oncol 2011; 18: 800-4.

Received: 04/05/2016 Accepted: 28/07/2016 